\title{
Laser Induced Breakdown Spectroscope on Chandrayaan-2 Rover: a miniaturized mid-UV to visible active spectrometer for lunar surface chemistry studies
}

\author{
A. S. Laxmiprasad*, R. V. L. N. Sridhar, Adwaita Goswami, K. A. Lohar, \\ M. V. H. Rao, K. V. Shila, Monika Mahajan, Bijoy Raha, T. S. Smaran and \\ B. Krishnamprasad \\ Laboratory for Electro-Optics Systems, Indian Space Research Organisation, Bengaluru 560058 , India
}

\begin{abstract}
Laser Induced Breakdown Spectroscope (LIBS) instrument flown in Chandrayaan-2 mission to the Moon, is one of the scientific instruments on the Pragyaan rover. It is primarily developed to carry out in situ investigations for the elemental composition study of lunar regolith and pebbles on the Moon surface in a previously unexplored high latitude area in the southern polar region. A pulsed laser source, a set of optical lenses and mirrors, an aberrationcorrected concave holographic grating and a linear detector, are the principal electro-optical accessories of the instrument. The developed LIBS is a lightweighted $(\sim 1.1 \mathrm{~kg})$ and low power consuming $(\leq 1.2 \mathrm{~W})$ compact instrument. This paper presents the system engineering and development aspects of the LIBS instrument along with results from environmental tests. Performance evaluation of the instrument during endto-end testing is satisfactory and within desired specifications. Details on ground calibration techniques used to evaluate the instrument capability are also presented.
\end{abstract}

Keywords: Chandrayaan-2 mission, laser-induced ablation, Moon, plasma emission, spectroscopy.

To understand solar system bodies like planets, Moon and asteroids, it is essential to determine their chemical composition. One of the most fundamental pieces of information about any planetary body is the elemental composition of surface materials, since such studies of a planet's surface or interior, permits characterizing the present/past conditions under which minerals were formed. Understanding the origin and evolution of our natural satellite, the Moon, has been a long-standing scientific goal. The surface of the Moon is blanketed by a thin layer of weakly-cohesive detrital materials (regolith or soil) comprising rock fragments, mineral grains and glass

*For correspondence. (e-mail: aslp@leos.gov.in) particles. The chemistry and mineralogy of the lunar soils primarily reflect the composition of the underlying bedrock. Significant insights on the lunar regolith formation can be obtained by comparing chemical compositions of soils from widely-separated landing sites or from geologically distinct areas around a single site. The elemental composition of soil is anticipated to vary in abundance across surface locations and also with soil depth.

Localized mineral mapping depends heavily on landers and rovers on planetary bodies and demands specialized instruments. Alpha proton X-ray spectrometer (APXS) and X-ray fluorescence instruments are often used for surface chemistry investigations in past and current space exploration missions ${ }^{1,2}$. Though these experiments have space heritage, some have extended requirements of long integration times (several hours) per measurement and proximity access to the sample (the sensor is taken close to sample or the sample is brought close to the instrument). Such limitations can hamper scientific return from a mission. There are clear advantages to techniques that could overcome such limitations and enhance scientific returns. Laser Induced Breakdown Spectroscopy $(\mathrm{LIBS})^{3,4}$, technique has unique potential as a planetary surface exploration tool ${ }^{5-9}$. The LIBS method is a spectro-chemical technique that can provide elemental composition of samples, regardless of their physical state. The laser is a fundamental tool for this technology, which produces the induced plasma from which information on the material composition through its atomic emission spectrum is obtained. Remote or close-range analysis is possible by this technique since the focused laser spot can be directed to a target at a considerable distance from the rover to produce the excited plasma. This method offers several advantages over conventional methods, namely: (i) rapid multi-element detection; (ii) sensitivity in partsper-million range; (iii) no or very little sample preparation; (iv) remote access to the sample; (v) top layer dust removal and (vi) limited depth analysis. To date, this 


\section{RESEARCH ARTICLES}

method been used for numerous applications in diverse fields such as mining, archaeology, painting analysis, metallurgy, military, avionics and space applications. 'Chemcam' onboard the 'curiosity' rover, is the first space-based LIBS instrument that was flown to Mars and is currently functional in providing remote sensing data on the composition and elemental abundances of Martian rocks and soils ${ }^{10,11}$. The second instrument that was built on the LIBS technique was flown to the Moon in India's second lunar mission, Chandrayaan-2, which was launched on 22 July 2019.

Chandrayaan- $2^{12,13}$, in addition to having an orbiter spacecraft as in Chandrayaan-1 mission ${ }^{14}$, will orbit the Moon in a circular orbit at an altitude of $100 \mathrm{~km}$. It carries a lander craft (Vikram), which will soft land at a pre-determined lunar site ${ }^{15}$ and deploy a six-wheel rover (Pragyaan) to perform investigations on the lunar surface in a previously unexplored high latitude region in the southern polar region. Various payloads are developed for accommodation on the orbiter spacecraft, the lander and the rover, to study the geochemistry and mineralogy of the Moon, which will aid the scientific community to unravel and further the current understanding of the evolutionary history of our natural satellite. The rover carries two scientific instruments, namely, the LIBS and the APXS for chemical analysis of the lunar surface. Design, development and testing of the LIBS instrument was undertaken by the 'laboratory for electro-optics systems (LEOS)', a key laboratory of the Indian Space Research Organisation.

The developed LIBS ${ }^{16,17}$ instrument at LEOS is the first one to be flown to the Moon to carryout in situ/closerange measurements on Moon surface. LIBS comprises a compact ( $\sim 0.027$ litres) nano-second pulse duration laser source emitting at $1540 \mathrm{~nm}$ wavelength with $0.4 \mathrm{MW}$ peak power, which further in combination with a focusing optics unit (FOU), produces the power density in the range of few $\mathrm{GW} / \mathrm{cm}^{2}$ to cause surface ablation and plasma generation. A two-fold path collection optics unit (COU), collects the light during plasma decay and an in-house developed spectrograph unit realized on flatfield spectroscopy technique ${ }^{18,19}$, spectrally disperses the collected light towards a one-dimensional linear charge couple device (CCD) that records the signal. The recorded signal is fed to a processing unit that processes and displays the data as a spectrum. The major constraints and challenges faced and overcome during instrument design and development phases are: low lunar surface temperatures that may range from $-60^{\circ} \mathrm{C}$ to $-90^{\circ} \mathrm{C}$ (depending on the landing latitude and season), predefined investigation height of $205 \mathrm{~mm}$, overall instrument width not $>80 \mathrm{~mm}$, weight of $\leq 1.2 \mathrm{~kg}$, operating temperature range of $-20^{\circ} \mathrm{C}$ to $+55^{\circ} \mathrm{C}$, storage temperature range of $-40^{\circ} \mathrm{C}$ to $+70^{\circ} \mathrm{C}$ and spectral resolution of less than a nanometer. System engineering and instrumentation aspects of the instrument are explained in detail in the next section. To validate the LIBS design and to ensure its successful operation onboard Chandrayaan-2 rover, several instrument-level and integrated tests with the rover are devised and performed. These tests include a performance evaluation plan to verify correct 'end-to-end' system operation, performance evaluation and instrument calibration. These tests are aided to provide information associated with operational peculiarities of the LIBS instrument, which is vital for the checkout of the instrument to rover interface. Two models of instrument are being developed, namely, QM (qualification model) and FM (flight model). The QM is used to qualify the unit for flight environment (as defined by ISRO) and later used for ground simulations, while the FM, subject to ISRO norms for acceptance is integrated into the FM rover. Calibration experiments employing diverse standard and certified reference soil powder samples that vary in elemental compositions were carried out under vacuum pressures of $\sim 2 \times 10^{-7}$ torr to investigate the elemental abundance determination capability of the instrument. Figure 1 depicts the three-dimensional Unigraphics (3D-UG) structural model of the rover along with the LIBS instrument that is depicted as mounted at the rover bottom chassis. Figure 2 shows the LIBS-QM unit. Salient features of the LIBS instrument are summarized in Table 1, whereas the sub-systems level engineering design parameters and specifications are listed in Table 2 in the later section.

\section{Science investigations}

The primary scientific objectives of LIBS instrument are to identify and derive abundance of elements that are

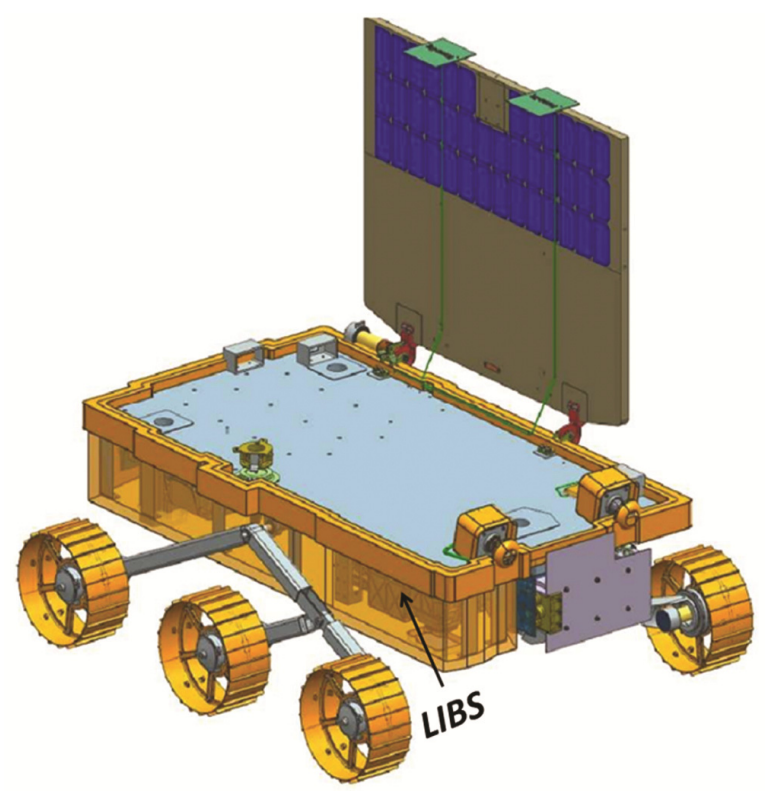

Figure 1. Three-dimensional unigraphics rover model with LIBS located beneath the chassis. 
Table 1. Salient features of the laser induced breakdown spectroscope instrument

\begin{tabular}{ll}
\hline Parameter & \multicolumn{1}{c}{ Specification } \\
\hline Instrument to surface distance (ITSD) & $205 \mathrm{~mm} \pm 8 \mathrm{~mm}$ \\
Pointing direction & Lunar surface \\
Angular field-of-view & $\pm 3^{\circ}$ \\
Spectral range & $220-800 \mathrm{~nm}$ \\
Resolution & $\leq 1 \mathrm{~nm}$ \\
Spot size (diameter) at ITSD & $\sim 100 \mu \mathrm{m}$ \\
Weight & $\sim 1.1 \mathrm{~kg}$ \\
& \\
Power & \\
$\quad$ & $1.19 \mathrm{~W}$ \\
$\quad$ Pteady state & $10.42 \mathrm{~W}($ for $20 \mathrm{shots}$ at $5 \mathrm{~Hz}$ repetition rate) \\
Volume $(L \times W \times H)$ & $180 \mathrm{~mm} \times 150 \mathrm{~mm} \times 80 \mathrm{~mm}$ \\
Operating temperature & $-20^{\circ} \mathrm{C}$ to $+55^{\circ} \mathrm{C}$ \\
Storage temperature & $-40^{\circ} \mathrm{C}$ to $+70^{\circ} \mathrm{C}$ \\
\hline
\end{tabular}

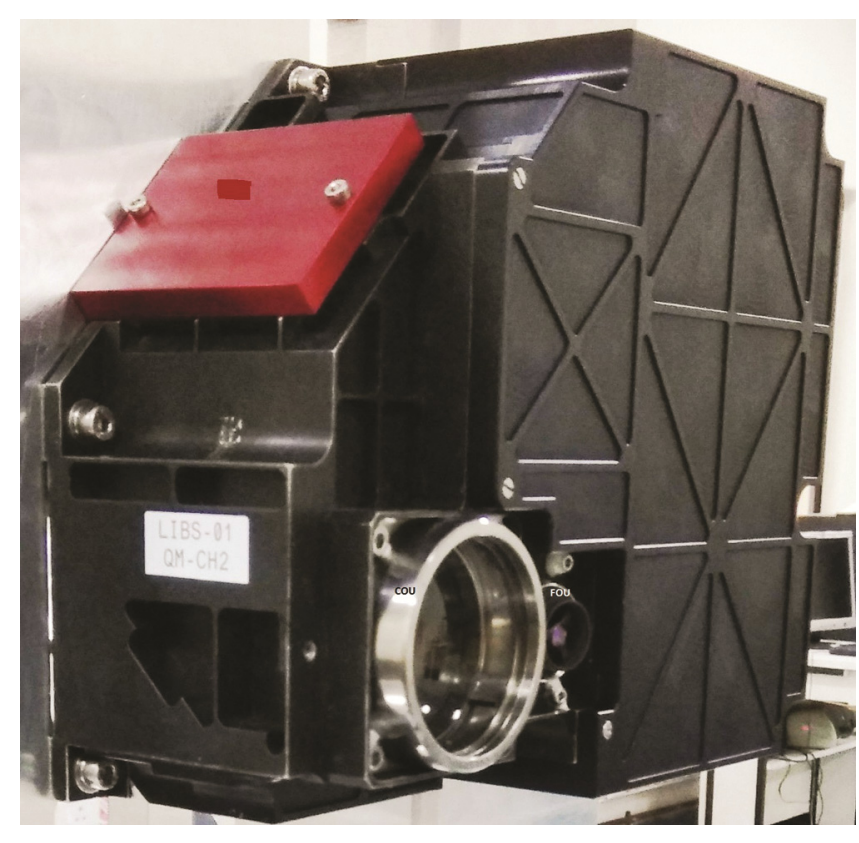

Figure 2. Developed LIBS-qualification model.

commonly found in lunar rock-forming minerals, i.e. O, $\mathrm{Na}, \mathrm{Mg}, \mathrm{Al}, \mathrm{Si}, \mathrm{K}, \mathrm{Ca}, \mathrm{Fe}, \mathrm{Cr}, \mathrm{Mn}$, Ti, etc. and the detection of volatile and trace elements $(\mathrm{H}, \mathrm{C}, \mathrm{N}, \mathrm{S}$ and $\mathrm{P})$ in the vicinity of the landing site. These observations could enable investigation of hydrates and organic content in sampling area by analysing $\mathrm{H}, \mathrm{C}, \mathrm{N}$ and $\mathrm{O}$ emission line characteristics. In addition to the above-mentioned science goals, LIBS measurements acquired by the rover at each mobility stop will address the local compositional heterogeneity. These measurements along with data from other instruments on Chandrayaan-2, can collectively aid in enhancing insight into the petrology at the landing site.

\section{Principle of operation and operational modes}

LIBS instrument built on the technique of laser-induced plasma generation, uses a focused laser pulse as the ener- gy source to ablate the sample. In this light-matter interaction process, the sample surface gets heated, melted, vapourized, atomized and ionized. An intense plasma plume that comprises excited atoms, ions, electrons and neutral atoms is generated as a result of high laser power deposition on the sample. After a short relaxation time of typically about few tens of microseconds ${ }^{20}$, these excited species will de-excite to lower energy states by emitting their characteristic electromagnetic radiation. The emitted electromagnetic radiation is collected and fed to a spectrometer that contains a dispersive element to diffract the received radiation into discrete wavelengths. The dispersed light then impinges onto a light-sensitive detector that yields the output response as a function of wavelength. Peak wavelengths of emission lines in the recorded spectrum disclose element identification, while the background subtracted line intensity aid in determination of the elemental abundance.

LIBS instrument can function in two operational modes, namely 'multiple shot capture (MSC)' and 'single shot capture (SSC)'. In 'MSC' mode, multiple laser shots impinge on the sample and the plasma emission is acquired either after the last laser shot or after each laser shot. Plasma emission capture after the last laser shot will aid in analysing dust covered samples, whereas the latter will be used to acquire multiple spectra at the same investigation site that help to improve the analytical precision. The 'SSC' mode is similar to 'MSC' mode except for the fact that here, the 'number of pulses' is always ' 1 '. In these two operational modes, plasma emission spectrum will be captured after the background signal acquisition (no-laser fire/dark light condition).

\section{Instrument overview and system engineering}

Theoretical estimations (related to elemental ablation kinetics and emissivity estimations) that drove the instrument baseline configuration are published elsewhere $^{21}$. We carried out studies related to estimation of 
Table 2. LIBS instrument design parameters and specifications

\begin{tabular}{|c|c|c|}
\hline Entity & Parameter & Value (SI units) \\
\hline \multirow[t]{5}{*}{ Laser source } & Type & Diode pumped solid state \\
\hline & Energy & $3-4 \mathrm{~mJ}$ \\
\hline & Pulse duration & $7 \mathrm{~ns}$ \\
\hline & Wavelength & $1540 \mathrm{~nm}$ \\
\hline & Beam diameter & $0.8 \mathrm{~mm}$ \\
\hline \multirow[t]{3}{*}{ Focusing optics unit } & Type & Fixed focus lens system \\
\hline & Lens material & N-BK7 \\
\hline & Focal length & $200 \mathrm{~mm}$ \\
\hline \multirow[t]{5}{*}{ Collection optics unit } & Type & 2-Fold lens-mirror geometry \\
\hline & Entrance pupil diameter & $37 \mathrm{~mm}$ \\
\hline & Application range & $220-800 \mathrm{~nm}$ \\
\hline & Material & Fused silica, $\mathrm{MgF}_{2}$ and sapphire \\
\hline & Optical gain & 0.65 \\
\hline \multirow[t]{9}{*}{ Spectrograph unit } & Type & Flat-field \\
\hline & Spectral range & $190-800 \mathrm{~nm}$ \\
\hline & $\mathrm{F} \#$ & 2.2 \\
\hline & Slit type & Air precision slit etched on a SS-thin metal sheet \\
\hline & Slit shape, dimension & Rectangular, $50 \mu \mathrm{m}(w) \times 500 \mu \mathrm{m}(l)$ \\
\hline & Grating type & Aberration corrected concave holographic \\
\hline & Detector type & UV-enhanced linear CCD \\
\hline & Number of pixels & 2048 \\
\hline & Pixel size & $14 \mu \mathrm{m}(w) \times 200 \mu \mathrm{m}(l)$ \\
\hline \multicolumn{2}{|c|}{ Main base structure material } & Mg-alloy \\
\hline \multicolumn{2}{|c|}{ Opto-mechanical mounts material } & Ti-alloy \\
\hline \multicolumn{2}{|c|}{ Tele-command interface } & 16-bit serial data \\
\hline
\end{tabular}

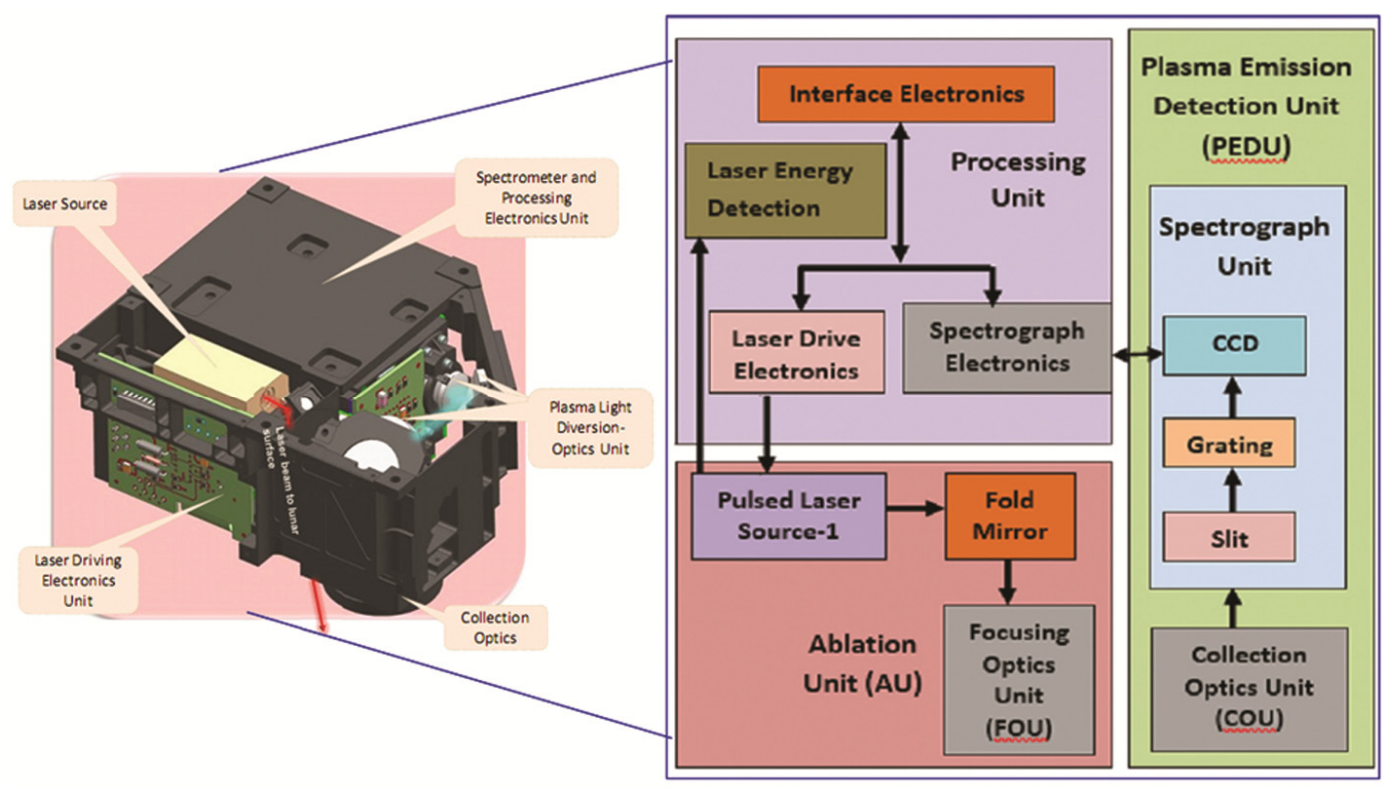

Figure 3. System architecture and block diagram.

minimum laser threshold intensity/power density for breakdown and vapourization, mass of the evaporated material, ablation depth, plasma emissivity, signal-to-noise ratio estimations and spot size requirement at the defined investigation distance, etc. These aided optimizations of the laser source, grating, detector and further guided the design of efficient focusing and collection optics modules for the LIBS instrument. Figure 3 presents the LIBS system architecture with the block diagram showing subsystems. As shown in Figure 3, the LIBS instrument suite consists of three major units: (i) Ablation Unit-AU, (ii) Plasma Emission Detection Unit-PEDU and (iii) Processing Unit-PU. The 'AU' comprises a compact laser source operating at $1540 \mathrm{~nm}$ wavelength with pulse 

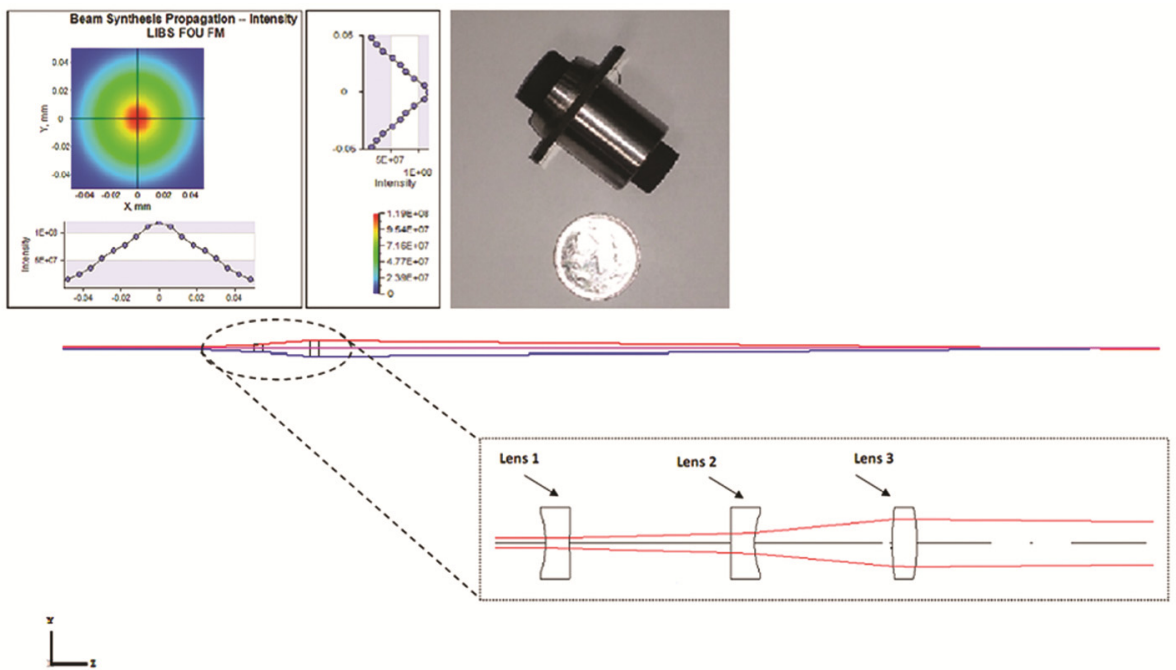

Figure 4. Ray trace diagram of focusing optics unit (FOU); inset figures: irradiance distribution within the resulted spot and developed FOU module.

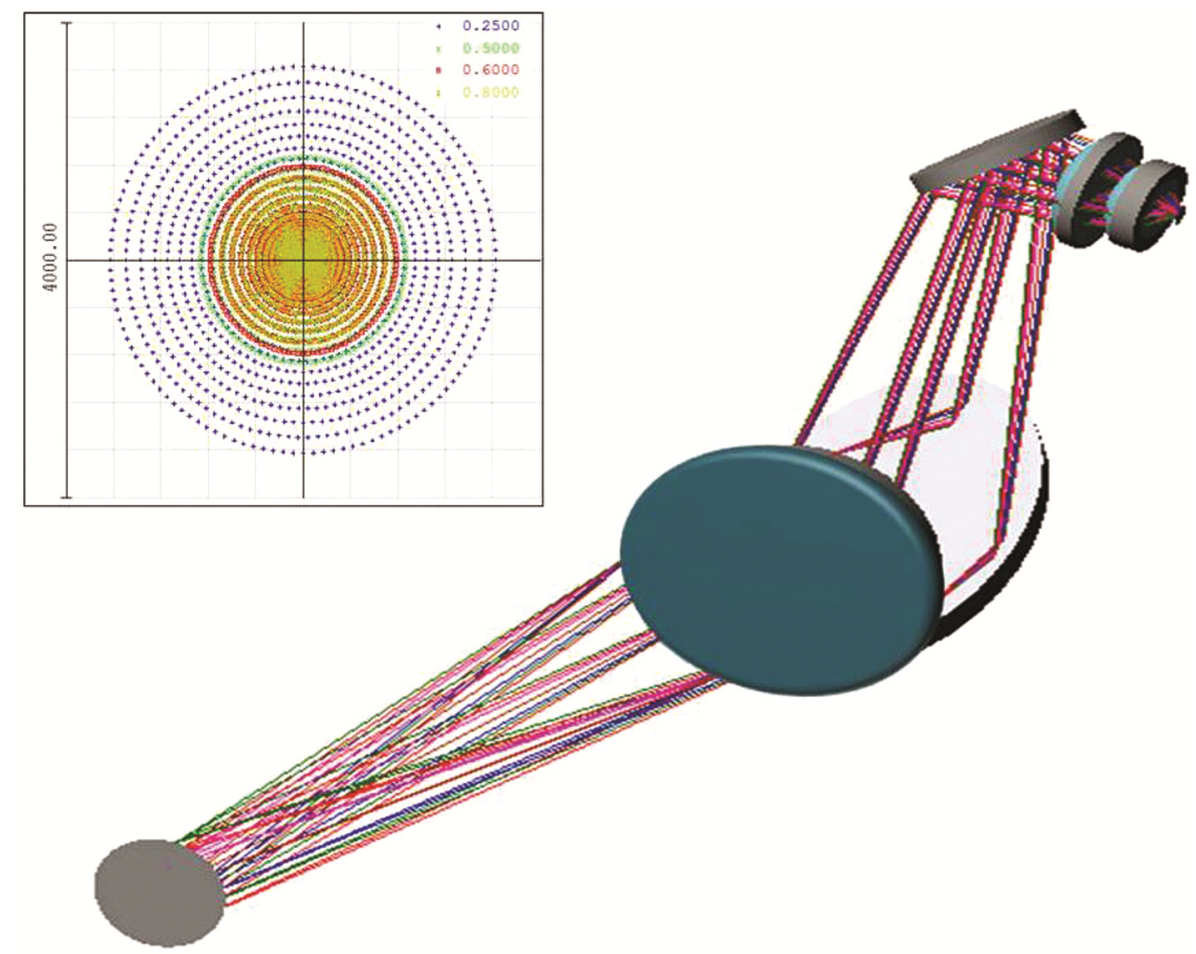

Figure 5. Ray trace diagram of collection optics unit (inset: resultant spot size at the image plane).

energy of $3.5 \mathrm{~mJ}$ and $7 \mathrm{~ns}$ pulse duration. This laser pulse is diverted to the FOU via a fold mirror to generate the desired power density $\left(\sim 6 \mathrm{GW} / \mathrm{cm}^{2}\right)$ for target surface ablation and plasma generation from a distance of $205 \mathrm{~mm}$. The 'PEDU' includes the COU (an optical unit having 3-lenses and 2-mirrors) and a spectrograph unit (SU). Light emission from the decaying plasma is collected by the COU and is analysed using a miniaturized, high resolution and high sensitive SU. The SU, is an indigenous development that was realized using an aberration-corrected concave holographic reflection grating (spectral range: $190-800 \mathrm{~nm}, \mathrm{~F} \# 2.2$ ), a rectangular slit (of width $50 \mu \mathrm{m}$ and length $500 \mu \mathrm{m}$ ) and a linear CCD as detector (2048 active elements). The 'PU' decodes the tele-command received from the rover, triggers laser drive electronics, captures and processes the CCD image. The realized PU, controls and monitors numerous operating and functional parameters such as pump pulse current, pulse repetition rate, number of pulses, integration time, delay time, mode of operation, 


\section{RESEARCH ARTICLES}

etc. in order to acquire effective spectral signatures from target surfaces. These three major units of LIBS instrument are housed to a black anodized main base structure, made of Mg-alloy. The most challenging aspect of the system design and engineering was to realize the fully integrated instrument within the available volume and permissible weight by the mission as mentioned in Table 1 and to meet the system performance requirements. Table 2 lists sub-systems wise design parameters and specifications of the LIBS instrument.

As shown in the ray trace diagram of the FOU in Figure 4, the first two lenses of FOU expand the incoming laser beam, whereas the third lens converge the expanded beam to the required spot size at the ITSD. Inset figures presented on the top-left hand side of Figure 4 show the irradiance (watts per $\mathrm{mm}^{2}$ ) distribution within the resultant spot size at the image distance along with developed FOU module. Figure 5 presents the ray diagram of the COU from which it is evident that the three lens elements serve the purpose of collection and focusing of the plasma emission at the entrance slit of the SU, while the two-fold path geometry-based mirror system aids in steering the collected radiation into the slit path. The inset picture of Figure 5 presents the spot diagram generated by the COU at the image plane. Figure 6 shows threedimensional mechanical structural model of the in-house developed SU having electronics cards wrapped around its supporting structure. As shown in the figure, the slit is positioned at the focus of the rear optics of COU and the $\mathrm{CCD}$ is placed in the focal plane of the grating. A 16-bit serial data command pattern is implemented in the processing logic unit to control diverse operational parameters and modes of LIBS instrument, suiting onboard operational requirements. Following the development of AU, PEDU and PU, the integrated LIBS system is subsequently tested and flight qualified.

\section{Performance and functional testing}

LIBS payload was subjected to functional, environmental (thermo-vacuum cycling test, thermal soak test, vibration test, shock test) and end-to-end tests both at the instrument and after integrating on to the rover.

\section{Functional testing}

Instrument response tests are carried out employing two different kinds of light sources; a discrete light emission source ( $\mathrm{Hg}-\mathrm{Ar}$ lamp) and a continuous emission source (a halogen lamp). Figure 7 shows the bench-top test-setup that was established to evaluate the integrated LIBS functionality and performance for the known source emission radiation characteristics. The test setup shown in Figure 7 has also been used for the spectrometric calibration of the system whose output aids in obtaining the correlation between pixel numbers and wavelengths. In addition to the above two tests, plasma emission spectrum from a thin aluminium sheet is captured and its spectral signature is compared from initial to final bench test phases. The achieved spectral resolution from these measurements is found to be $0.78 \pm 0.2 \mathrm{~nm}$ and the instrument is observed to be functional for the desired spectral band of $220-800 \mathrm{~nm}$.

\section{Environmental testing}

LIBS instrument functionality and performance are further evaluated by subjecting the payload to rigorous environmental test conditions. Figure 8 shows the cartoon diagram of the realized test set-up to evaluate the performance during thermo-vacuum cycling test phase. As shown in the figure, the integrated instrument is fixed to a $300 \mathrm{~mm}$ length vertical stainless-steel rod and mounted onto a common metallic base plate comprising a motorized $x y$-translation stage equipped with a sample holder

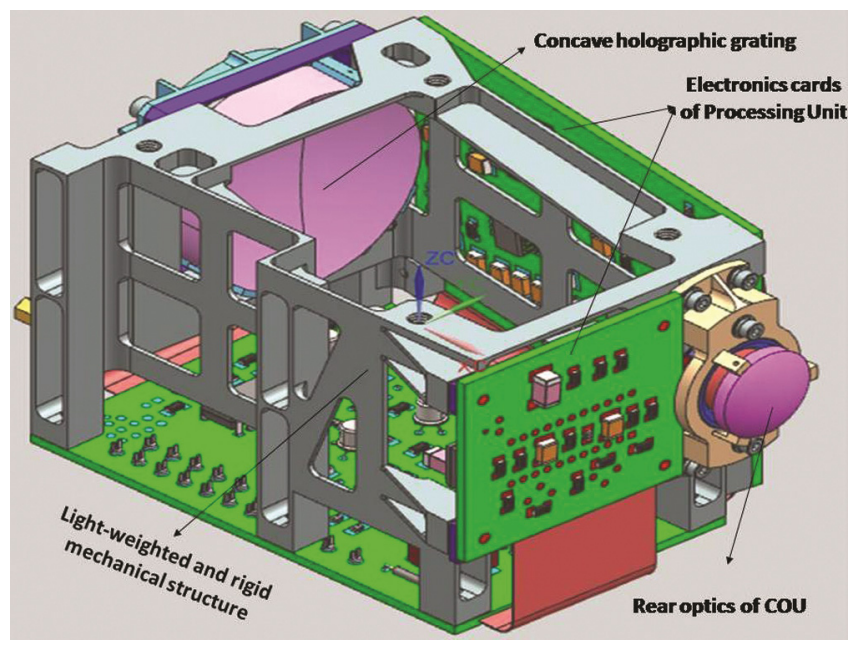

Figure 6. 3D-Mechanical structural design of spectrograph unit.

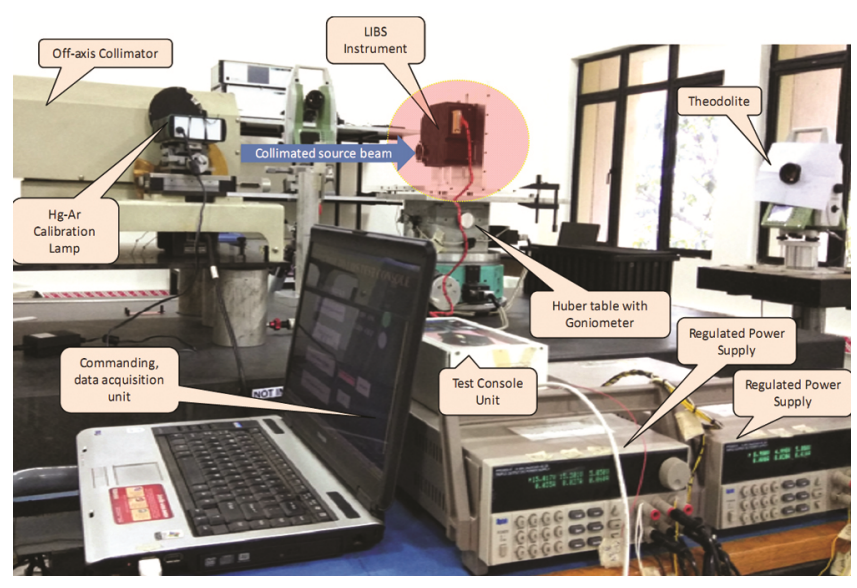

Figure 7. Spectrometric calibration test set-up.

CURRENT SCIENCE, VOL. 118, NO. 4, 25 FEBRUARY 2020 


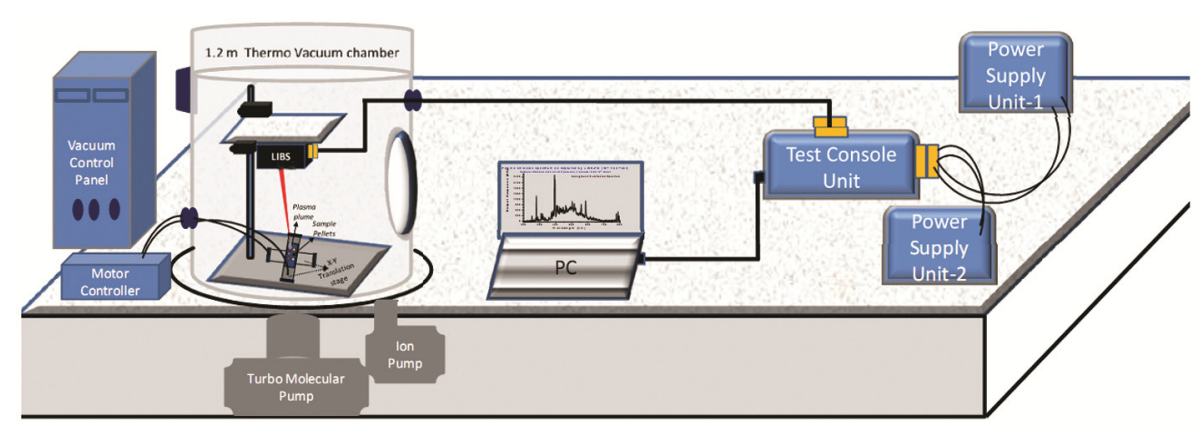

Figure 8. Cartoon diagram of performance evaluation test set-up during thermo-vacuum cycling phase.
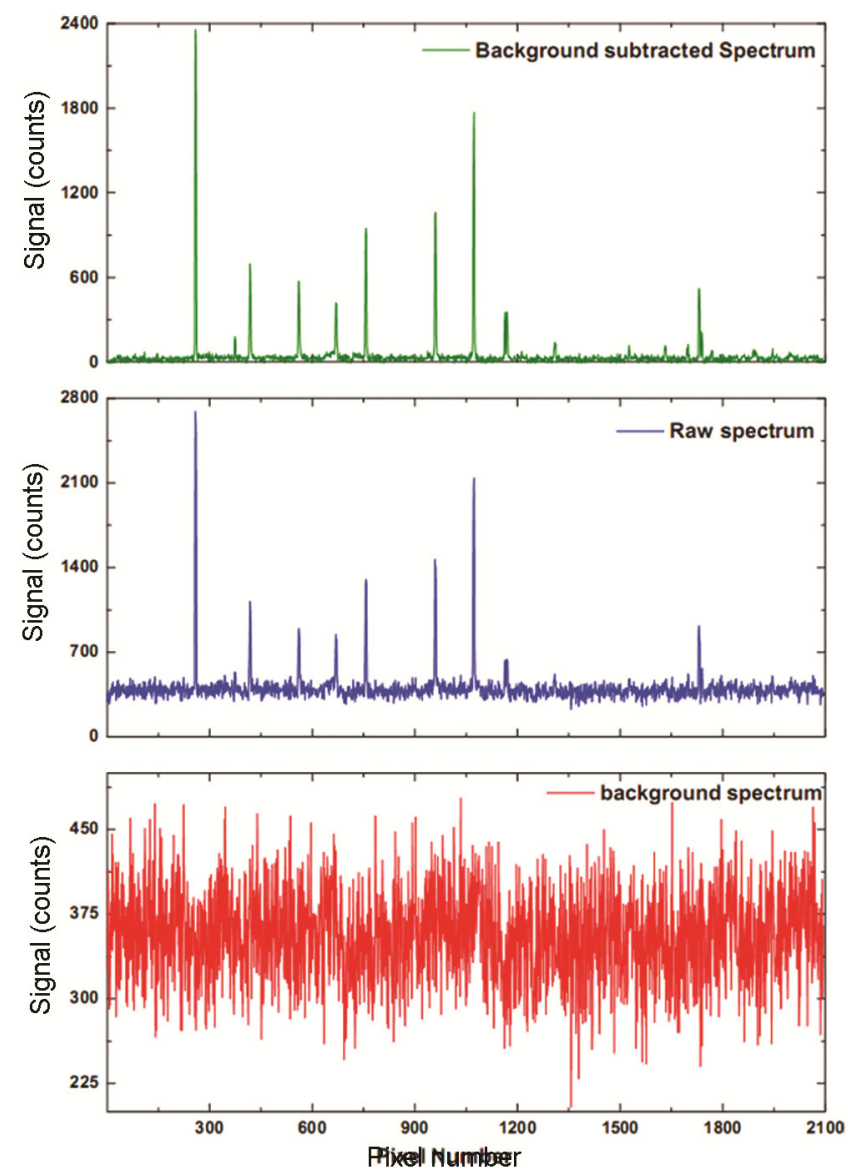

Figure 9. $\mathrm{Hg}-\mathrm{Ar}$ source emission spectrum as recorded by the LIBS (red: background, blue: unprocessed data; green: background subtracted data).

plate. Pressed powder discs of soil samples are placed in the sample holder for performance evaluation in vacuum environment. The instrument is powered $\mathrm{ON}$ continuously for several thermal cycles at $-20^{\circ} \mathrm{C}$ and at $+55^{\circ} \mathrm{C}$ with cycle durations of $2 \mathrm{~h}$ (short cycle) and $24 \mathrm{~h}$ (long cycle). To prevent corona and to ensure reliable operation, the instrument was soaked in vacuum $\left(3 \times 10^{-5}\right.$ torr $)$ prior to power ON. Measured functional and performance test parameters are well within specification tolerance limits.

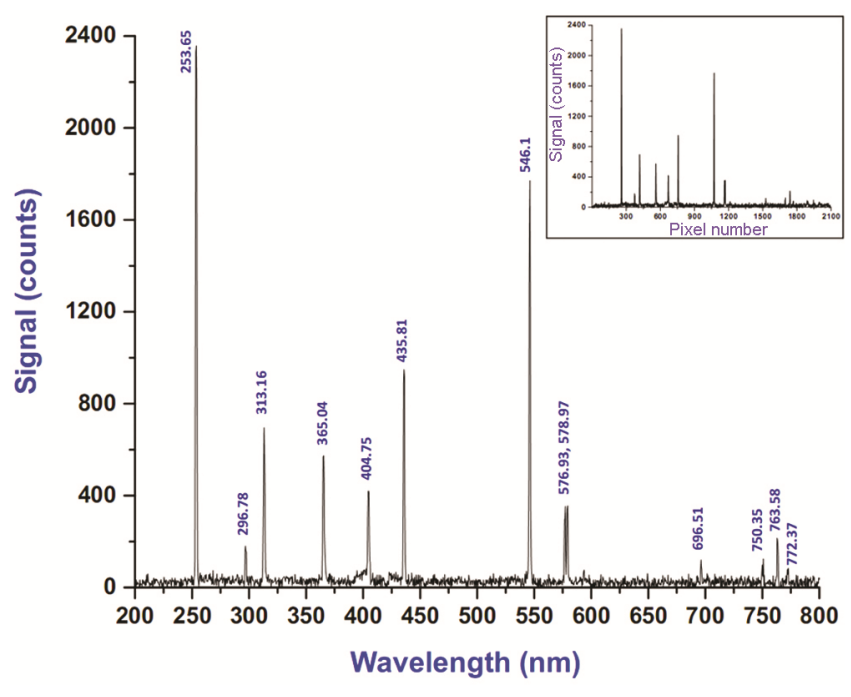

Figure 10. Spectrometric calibration generated $\mathrm{Hg}-\mathrm{Ar}$ source emission spectrum (inset: raw data spectrum).

Instrument is subjected to vibration for qualification levels, which involve mechanically induced frequency sweeps at levels of $18.1 \mathrm{~g}$ in $x, y$ and $z$-axis (random vibration) and $20 \mathrm{~g}$ in $x, y$ and $z$-axis (sine vibration). The LIBS-QM unit is further subjected to the 'shock test' for $700 \mathrm{~g}$ amplitude level in frequency range of 1000$5000 \mathrm{~Hz}$ in all 3-axes. Post shock test performance is satisfactory. After the completion of functional and environmental tests, LIBS is integrated to the rover and tested successfully for mechanical, electrical and data interfaces.

\section{Pre-flight calibration and data processing}

LIBS instrument calibration addresses qualitative and quantitative aspects. As shown in Figure 7, the spectral calibration of the instrument is carried out by acquiring the radiation emitting from a standard $\mathrm{Hg}-\mathrm{Ar}$ calibration lamp, whose spectral signature and peak emission wavelengths are known. The initial lamp spectrum that was acquired by the LIBS is a plot of instrument response versus pixel number. Peak centroids (pixel numbers) of emission peaks of the acquired lamp spectrum and known 


\section{RESEARCH ARTICLES}

peak emission wavelengths from the lamp reference spectrum, are used to find correlation factors to convert the pixel number into wavelength. Multiple regression analysis method was adopted to find correlation factors to relate the pixel number to wavelength. Figure 9 shows a ladder plot comprising the dark spectrum, the raw data spectrum (not corrected for background) as captured by the LIBS and the processed lamp emission spectrum corrected for the background. Figure 10 shows the postspectral calibration processed lamp emission spectrum as a function of wavelength (inset shows the uncalibrated spectrum). Calibration experiments to address elemental abundance estimation are carried out employing nearly 60 standard/certified reference materials and chemical analogues to lunar soil in a 0.25 metre ultra high vacuum chamber. A consistent database was generated from these experiments and calibration curves for selected elements of interest were generated to retrieve abundance. Figure 11 presents the background subtracted and baseline corrected plasma emission spectrum (captured in vacuum

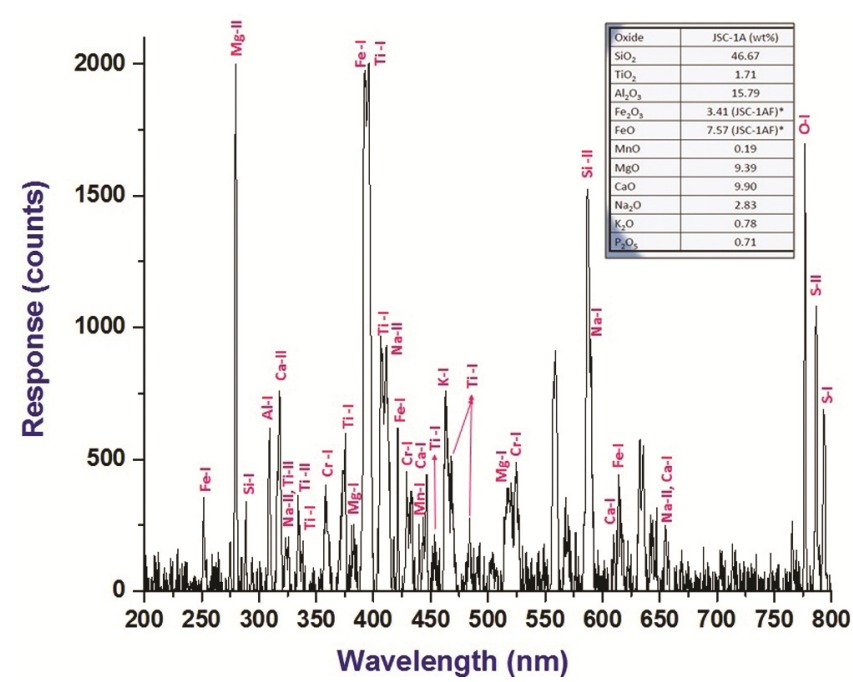

Figure 11. LIBS spectrum of JSC-1A sample pellet with elements detected.

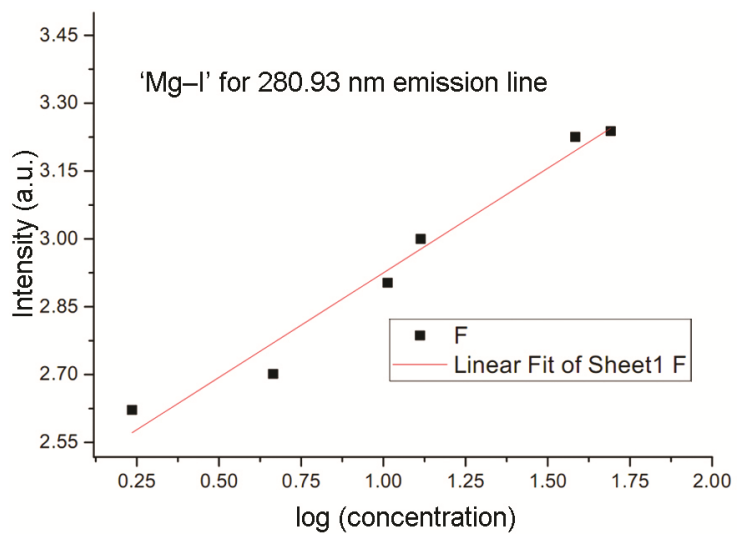

Figure 12. Calibration curve of ' $\mathrm{Mg}-\mathrm{I}$ ' for $280.93 \mathrm{~nm}$ emission line. pressure of $\sim 3 \times 10^{-10}$ torr) of the pressed powder disc made from the lunar regolith simulant (JSC-1A) ${ }^{22}$ sample. Labels of emission peaks in the spectrum represent elements that were detected, while 'I' and 'II' indices mentioned next to the element name indicates the ionization state of the element (I for neutral atomic state, II for 1st ionization state and so on). Qualitative analytical capability of LIBS instrument has been cross-verified with the data obtained from another analytical technique, i.e. the energy dispersive X-ray spectroscope and comparison of data statistics have shown high elemental identification capability of LIBS instrument. Figure 12 shows the calibration curve generated for the element 'magnesium' at peak emission wavelength of $280.93 \mathrm{~nm}$ along with the theoretical fit realized by means of a univariate analytical approach (i.e. $\log -\log$ linear regression). The maximum percentage of error obtained in abundance estimation is 5 .

\section{Conclusion}

The LIBS instrument developed based on the laserinduced plasma spectroscopy technique is the second one flown onto any other planetary body and would be the first one on the Moon. This instrument, in particular, is suited to carryout in situ chemical analysis of planetary surfaces owing to its simultaneous multi-element rapid detection capability and thus enhanced efficiency in the limited operational lifetime of a rover. The combined requirement of operating an instrument in an ultra-high vacuum environment and low temperature conditions and other design constraints, has contributed to the complexity of the system engineering and design. The developed instrument has been successfully qualified for space use; functionality and performance has matched the desired specifications in all aspects and is well calibrated on ground following the standard techniques and procedures. Performed experiments on geochemical standard and certified reference material samples in ultra-high vacuum environment and adopted data analytical approaches have shown high elemental detection capability. The maximum percentage of uncertainty in abundance estimation is $\leq 5 \%$. Application of other data analytical approaches like PLS, CF-LIBS, etc. are currently in progress to improve the quantitative analytical accuracy.

1. Reider, R. et al., The chemical composition of Martian soil and rocks returned by the mobile alpha proton X-ray spectrometer: preliminary result from the X-ray mode. Science, 1997, 278(5344), 1771-1774.

2. Priestley, T., Clark, B. C., Baird, A. K., Keil, K. and Rose, H. J., Preliminary results from the viking $\mathrm{X}$-ray fluorescence experiment: the first sample from Chryse Plantia, Mars. Science, 1976, 194(4260), 81-84.

3. Radziernski, L. J., Loree, T. R., Cremers, D. A. and Hoffman, N. M., Time-resolved laser-induced breakdown spectroscopy (LIBS): 
a new method for spectrochemical analysis. LANL Report, LAUR-82487, Los Alamos National Laboratory, NM, 1982.

4. Fang, Y. Y., Jagdish, P. S. and Hansheng, Z., Laser-induced breakdown spectroscopy, elemental analysis. Ency. Anal. Chem. (ed. Meyers, R. A.), Copyright @ John Wiley, 2000, 0066-2087.

5. Knight, A. K., Scherbarth, N. L., Cremers, D. A. and Ferris, M. J., Characterization of laser-induced breakdown spectroscopy (LIBS) for application to space exploration. Appl. Spectrosc., 2000, 54, 331-339.

6. Wiens, R. C., Arvidson, R. E., Cremers, D. A., Ferris, M. J., Blacic, J. D., Seelos IV, F. P. and Deal, K. S., Combined remote mineralogical and elemental identification from rovers - field and laboratory tests using reflectance and laser-induced breakdown spectroscopy, J. Geophys. Res. (Planets), 2002, 107, 8003; doi:10.1029/2000JE001439.

7. Colao, F. et al., Investigation of LIBS feasibility for in situ planetary exploration: an analysis on martian rock analogues. Planet. Space Sci., 2004, 52, 117-123.

8. Jobin Antony, K., Gurneesh, S. J., Nilesh Vasa, J., Sridhar Raja, V. L. N., Laxmiprasad, A. S., Modelling of laser-induced breakdown spectroscopy for very low pressure conditions, Appl. Phys. A, 2010; doi.org/10.1007/s00339-010-5782-1.

9. Lasue, J. et al., Remote laser induced breakdown spectroscopy (LIBS) for lunar exploration. J. Geophys. Res. Planets, 2012, 117, 1; https://doi.org/10.1029/2011JE003898.

10. Wiens, R. C. et. al., Chemcam science objectives for the mars science laboratory (MSL) rover. Lunar Planet. Sci. XXXVI, 2005 , p. 1580.

11. Wiens, R. C., Maurice, S. and the ChemCam team, The ChemCam instrument suite on the Mars science Laboratory Rover curiosity: remote sensing by laser-induced plasmas. Geochem. News, 2011, 145, $41-48$.

12. Mylaswamy Annadurai, Alex, T. K., Krishnan, A. and Rama Murali, G. K., Chandrayaan-2 mission: India's first soft landing mission onto Moon, 63rd International Astronautical Congress, 2012, 12634.

13. Venkatesan Sundararajan, Overview and technical architecture of India's Chandrayaan-2 mission to the Moon. AIAA Aerospace Sciences Meeting, AIAA SciTech Forum, 2018, p. 2178; https://doi.org/10.2514/6.2018-2178.

14. Goswami, J. N. and Annadurai, M., Chandrayaan-1: India's first planetary science mission to the moon. Curr. Sci., 2009, 96, 486491.

15. Amitabh, K., Suresh and Srinivasan, T. P., Potential landing sites for Chandrayaan-2 lander in southern hemisphere of Moon, 49th Lunar and Planetary Science Conference, 2018, 1975.
16. Sridhar Raja, V. L. N., Laxmiprasad, A. S., Adwaita Goswami, Lohar K. A. and Rao, M. V. H., Surya Menon and Kamalakar, J. A., Design, development and performance facets of a prototype laser induced breakdown spectroscope (LIBS) instrument for Chandrayaan-2 rover, 62nd International Astronautical Congress, 2011, IAC-11.A3.2A.10.

17. Laxmiprasad, A. S., Adwaita Goswami, V. L. N. Sridhar Raja, Lohar, K. A., Rao, M. V. H., Monika Mahajan and Bijoy Raha, The LIBS instrument for Chandrayaan-2 rover: engineering model development aspects, 67th International Astronautical Congress, 2016, IAC-16.A3.2B.8.

18. Lerner, J. M., Chambers, R. J. and Passereau, G., 'Flat field imaging spectroscopy using aberration corrected holographic gratings. Proceedings of the. SPIE 0268, Imaging Spectroscopy I, 1981; doi.org/10.1117/12.959934.

19. Qian Zhou, Jinchao Pang and Kai Ni, A portable flat-field concave grating spectrometer with high resolution. Proceedings of the SPIE 9271, Holography, Diffractive Optics, and Applications VI, 2014, 92711K; doi.org/10.1117/12.2072035.

20. Miziolek, A., Palleschi, V. and Schechter, I., Laser-Induced Breakdown Spectroscopy (LIBS) Fundamental and Applications, Cambridge University Press, New York, 2006.

21. Laxmiprasad, A. S., Sridhar, Raja V. L. N., Surya Menon, Adwaita Goswami, Rao, M. V. H. and Lohar, K. A., An in situ laser induced breakdown spectroscope (LIBS) for Chandrayaan-2 rover: ablation kinetics and emissivity estimations. Adv. Space Res., 2013, 52, 332-341; http://dx.doi.org/10.1016/j.asr.03.021.

22. Ray, C. S., Reis, S. T., Sen, S. and Dell, J. S. O., JSC-1A lunar soil simulant: characterization, glass formation and selected glass properties. J. Non-Cryst. Solids, 2010, 356(44-49), 23692374.

ACKNOWLEDGEMENTS. We thank Shri A. S. Kiran Kumar (former Chairman, ISRO), Dr T. K. Alex, Mr. J. A. Kamalakar and Dr G. N. Rao (founder and former Directors, LEOS) for their valuable inputs and timely suggestions offered in periodical reviews during the course of instrument development realization. We thank other engineers and technicians of LEOS, who involved significantly in design, fabrication and coating of optical elements; PCB layout designs and components wiring of electronics cards; alignment, assembly and integration activities; environmental tests, etc.

Received and accepted 27 August 2019

doi: $10.18520 / \mathrm{cs} / \mathrm{v} 118 / \mathrm{i} 4 / 573-581$ 